\title{
Thermal and Mechanical properties of epoxy-jute fiber composite
}

\author{
H. Ahmad, M. A. Islam, M. F. Uddin \\ Department of Chemical Engineering and Polymer Science (CEP), Shahjalal University of Science and Technology, \\ Sylhet-3114. E-mail: humayun_ceps2007@yahoo.com
}

\begin{abstract}
Chopped jute fiber-epoxy composites with varying fiber length (2-12 mm) and mass fraction (0.05-0.35) had been prepared by a heat press unit. The cross-linked product was characterized in terms of specific gravity, thermal conductivity, tensile strength, Young modulus and elongation at break. The transverse thermal conductivities for randomly oriented fibers in the composite were investigated by Lees and Charlton's method. The tensile strength, Young modulus and elongation at break were investigated by a Universal Tensile Tester. With an increase in the fiber content (irrespective of the fiber length), the thermal conductivity of the composite decreases; the decreasing rate being highest for the fiber length of $2 \mathrm{~mm}$ followed by that for the fiber length of 6 and $12 \mathrm{~mm}$. The decreasing rate of the thermal conductivity of the jute-epoxy composite is comparatively higher to that reported in literature for acrylic polymer hemp fiber composite. The tensile strength also decreases with the increase of the fiber content in the composite. The fiber length does not show to have significant effect on the tensile strength of the composite; the variation in strength being masked within experimental error. The Young modulus increases with the increase of fiber content within elastic limit; showing the highest values for the fiber length of $6 \mathrm{~mm}$ followed by those for the fiber length of $2 \mathrm{~mm}$ and $12 \mathrm{~mm}$. The elongation at break shows slightly increasing trend up to $15 \%$ fiber content, but beyond that it decreases drastically. The specific gravity decreases with the increase in the fiber content and thus the recalculated specific tensile strength is found to keep at a stable level of $36 \mathrm{MPa}$ up to the fiber content of $20 \%$, and beyond that the specific tensile strength decreases with the increase in the fiber content. It is concluded that jute fiberepoxy composite could be used as a good heat-insulating material. Further investigation is recommended on the improvement of the thermal insulation keeping the mechanical properties unchanged or even improved. The TGA study is also required to ascertain the field of application of the material.
\end{abstract}

\section{INTRODUCTION}

Various products are produced from polymer materials. The properties of these materials can be tailored with the incorporation of fillers, fibers and other additives. In the last decades, intensive research has been conducted on the improvement of the natural fiber reinforced polymer composites (NFRPC). As a result, polymer composites have been widening their range of application in various fields. Nowadays, applications of NFRPCs can be found in housing construction material, industrial objects, storage devices, furniture, electric devices, thermal insulations and automotive parts [1]. Rapid growth of NFRPC technology is attributed mainly to the low cost of the fiber and the ecological benefits. Besides that, the natural fibers have some other advantages over synthetic reinforcing fibers such as biodegradability, renewability, availability in abundance, low specific gravity, non-abrasiveness and ease of surface modification.

Among the natural fibers, most studied as the reinforcing ingredient in polymer composites are flax, hemp , jute, sisal, kenaf, coir, kapok, banana, henequen, oil palm, bamboo, wheat and waste silk fibers [2-6]. Most of the studies are concerned with the improvement of the tensile properties (tensile strength, modulus of elasticity and elongation at break) of the composites [7]. As the field of application of the composites widens, others properties such as thermal and electrical properties, sound and vibration dissipation properties, sorption and permeation of gas and vapors, stability towards mechanical, electrical and thermal shocks etc are equally important and deserve proper attention. Scientific investigation on these properties of the NFRPCs is still limited. In an effort to contribute in these aspects, researches on the thermal properties of NFRPCs have been initiated in the laboratory of material science at the Department of CEP.

Investigations on the thermal properties of NFRPCs are scarce [8-9]. Behzad and Sain [9] reported that the thermal conductivity of hemp fiber- acrylic polymer composite in the transverse direction decreased as the volume fraction of fibers increased. On the other hand for in-plane direction, the thermal conductivity of oriented composites enhanced with increasing the volume fraction of fiber. To validate the experimental results, the heating tests for the thermal conductivity measurements were simulated by a finite element model using the thermal conductivity values obtained from the experiments. The predicted temperatures showed close agreement with the measured ones. Mangal et al. [10] also observed that the increasing the pineapple leaf fiber content in the matrix decreased the thermal conductivity and thermal diffusivity of the composite. Osugi et al. [8] studied the Manila hemp fiber reinforced poly lactic acid and epoxy resin composite and also confirmed that 
the thermal conductivity of the composite decreased with increasing fiber content.

Sweeting et al. [11] developed a new experimental method to determine the in-plane and though-thickness thermal conductivities of polymer matrix composites. The transient temperature gradient in the given direction was recorded using thermocouples and the result was processed numerically by an inverse approach to determine the directional thermal conductivity. The validation of the method was conducted using composites with known thermal properties, and excellent correlation was found between the known and determined thermal conductivities. Agrawal et al. [12] studied the thermal conductivity and thermal diffusivity of oil-palm-fiber-reinforced untreated and differently treated composites using transient plane source technique at room temperature. They concluded that all the silane and alkali treatments of the fibers increased the thermal conductivity and thermal diffusivity of the composites in comparison with the acetylated composite. Kalaprasad et al. [13] analyzed and presented the thermal conductivity and thermal diffusivity of sisal-, glass-, and sisal-glass hybrid fiber reinforced polyethylene composites at high temperatures and with different fiber orientations. The difference between the thermal conductivity properties in parallel and perpendicular directions with respect to fiber length was the highest for sisal-fiber reinforced polyethylene as a result of the anisotropic nature of the fiber.

Among the natural fibers, jute is one of the most important plant based fibers for composite materials due to its moderate specific strength, stiffness, low density, less abrasive behavior to the processing equipment, good dimensional stability and harmlessness. It is abundant in Bangladesh and the country has the potentiality to increase its production, if the market demands that. In the past few years several studies have been done on the potential use of jute fiber as reinforcing materials in a number of polymers such as polyester, polypropylene, polyethylene and natural rubber. Some investigations were conducted on the jute fiber reinforced epoxy composites, but they are also related basically to mechanical properties of the composite [14-17].

Epoxy resin is well-known for its excellent adhesion capability to materials of different nature including fiber materials. It has low shrinkage upon curing, good chemical resistance. Epoxies have superior mechanical and electrical properties, thermal insulation and good performance at elevated temperatures. Thus, investigations into the thermal properties of the jute fiber reinforced epoxy composite parallel to the study of the tensile property bear immense importance in connection with the potential application of the composite as thermal insulator in various applications such as refrigerated containers, building panels, fire walls and doors.

The purpose of the present work was to prepare epoxyjute composite with, and to study the effect of fiber length and mass fraction on the thermal conductivity and tensile properties of the composite. It is found that the thermal conductivity decreases, but the tensile strength and the modulus of elasticity increase with the increase in the fiber content in the composition. The fiber length does not have significant effect on the tensile properties, but slightly affect the thermal conductivity. This is a mere initiation of systematic research on the less studied polymer-fiber composite-properties such as thermal, electrical and vibration-damping parallel to improving the biodegradability, mechanical strength and specific gravity. The first results in this direction are stimulating for further research.

\section{EXPERIMENTAL}

\subsection{Material}

Jute fiber was used as a reinforcing phase and the epoxy resin (polycondensation product of epichlorohydrin with bis-phenol A) was used as a matrix phase. Both were collected from local market. Tetraethylenetriamine was used as the hardener. Wax used as a mould releasing agent.

\subsection{Methods}

2.2.1 Composite preparation: Untreated jute fibers were chopped into average lengths of $2 \mathrm{~mm}, 6 \mathrm{~mm}$ and $12 \mathrm{~mm}$. For all types of fiber-length, the composites were prepared with 5- $35 \mathrm{wt} \%$ of fiber. The chopped jute fibers were dried at $100^{\circ} \mathrm{C}$ for $1 \mathrm{hr}$ to remove moisture. Different proportion of untreated chopped jute fibers of different length-type was mixed with the resin composition with epoxy-hardener ratio of 10:1. For each length-type seven compositions were prepared with the jute fiber content in the range of 5-35 wt $\%$. The mixture was homogenized with manual stirring. Then the mixture was placed in a mold.

Three different types of mold were used for the preparation of composite samples, which were with the dimensions of $120 \times 120 \times 2 \mathrm{~mm}^{3}, 120 \times 120 \times 6 \mathrm{~mm}^{3}$ and $120 \times 120 \times 6 \mathrm{~mm}^{3}$.

Then the mold was placed on a hydraulic press unit (CARVER, Model-3853-0, S/N-12000-944, USA) at room temperature. A moderate pressure (45psi) pressure was applied to prevent the air entrapped in the liquid composition. The final composite was released from the press unit after 24 hours. Then it was placed in an oven at $100^{\circ} \mathrm{C}$ for $1 \mathrm{hr}$ for curing and the cured samples are stored in polyethylene bags to keep them humid-free.

2.2.2 Specimen for tensile test: For the measurement of tensile properties, the test specimens were cut from the hardened composite yielding them the form of a rectangular parallelepiped with the length 60$80 \mathrm{~mm}$, width $17-27 \mathrm{~mm}$ and thickness $2-6 \mathrm{~mm}$. 
2.2.3 Specimen for conductivity tests: For transverse thermal conductivity measurements, the test specimens were cut from the hardened composite yielding them the form of dense-circular wheels with the diameter of $51 \mathrm{~mm}$ and average thickness of $2 \mathrm{~mm}$, $4 \mathrm{~mm}$ and $6 \mathrm{~mm}$.

\subsection{Experimental methods}

\subsubsection{Measurement of thermal conductivity:}

Thermal conductivity was measured by Lees and Charlton's apparatus. The test specimen is placed between two metallic discs. The upper disc is heated by electricity and this heat is transferred to lower disc through test specimen by conduction mechanism. The lower disc is open to air and loses heat by convection and radiation mechanism. The temperature of the upper and the lower disc (denoted as $T_{U}$ and $T_{L}$ respectively) gradually increases until a steady state is attained, and the corresponding temperature of the upper and lower disc (denoted as $T_{U s}$ and $T_{L s}$ respectively) is recorded. At this condition the heat flux $q_{c o n}$ passing through the test specimen by conduction is equal to the rate of heatloss $q_{c v, r}$ by the lower disc by convection and radiation to the surrounding air. It is assumed that the thermal conductivity of the metal discs are much higher than that of the sample such that the resistance to thermal energy flux lies solely depends on the sample. Thus there is no temperature gradient in the discs at steady state and the temperature of the discs is the same as that of the sample-surfaces they are in contact with. Under such condition, the thermal conductivity $K(\mathrm{~W} / \mathrm{m} . \mathrm{K})$ is calculated from the heat balance equation (Eq. 1). Thus,

$$
\begin{aligned}
& q_{c o n}=q_{c v, r} \Rightarrow \\
& K A\left(T_{U s}-T_{L s}\right) / l=\left.m s\left(-d T_{L} / d t\right)\right|_{T_{L}=T_{L s}}
\end{aligned}
$$

Where $K(\mathrm{~W} / \mathrm{m} . \mathrm{K})$ is the thermal conductivity, $A\left(\mathrm{~m}^{2}\right)$ and $l(\mathrm{~m})$ are respectively the cross section area and the thickness of the sample, and $m(\mathrm{~kg})$ and $s\left(\mathrm{~J} / \mathrm{kg} .{ }^{\circ} \mathrm{C}\right)$ are respectively.

The rate of cooling (heat loss) $q_{c v, r}$ by the lower disc at $T_{L}=T_{L S}$ is determined in a separate experiment. The temperature of the lower disc $T_{L}$ is raised gradually to $10^{\circ} \mathrm{C}$ above from the recorded steady state temperature $T_{L s}$ through another heating arrangement at the upper disc and the heating process is interrupted by leaving the system cool down naturally. The falling of temperature of the lower disc $T_{L}$ is recorded until the temperature falls down by about $10^{\circ} \mathrm{C}$ below $T_{L s}$. Then a temperature $T_{L}$ vs time $t$ curve is plotted. The tangent on the curve at $T_{L}=T_{L S}$ is drawn. The slope of the curve $d T_{L} / d t$ at $T_{L}=T_{L s}$ is put in Eq. (1) to calculate the value of $K$. For details about the method, the readers are referred to [18]
2.3.2 Measurement of tensile properties: The tensile strength and the elongation at break were measured by a tensile testing instrument (EPOCH, Model-LSZ-52, and Range: 0-500 kg) at a deformation rate of $14.6 \mathrm{~mm} / \mathrm{min}$. Three test specimens from every composition were tested and the mean value was recorded. The average modulus of elasticity is calculated from the stress-strain curve in strain range of $0-0.18$.

\subsubsection{Measurement of specific gravity (bulk):}

The specific gravity (bulk) $\gamma_{b}$ of the composite is defined as the ratio of the bulk density $\rho_{b c}$ of the composite to that of water $\rho_{w}$. For the purpose, specimens were cut in rectangular shape and weighed. The geometric dimensions (length $L$, breadth $b$ and width $w$ ) were also measured. The value of $\gamma_{b}$ is calculated by the following formula:

$\gamma_{b}=\rho_{b c} / \rho_{w}=m /\left(\operatorname{Lbw} \rho_{w}\right)$

Where, $\mathrm{m}$ is the mass of the rectangular specimen.

\section{RESULTS AND DISCUSSION}

\subsection{Effect of composition on the thermal conductivity}

\subsubsection{Determination of thermal conductivity:} The thermal conductivity was measured through transverse direction. The mode of temperature increase at the two faces of the circular composite specimen is presented in Fig3.1. The data for other specimens are similar (not shown for brevity).

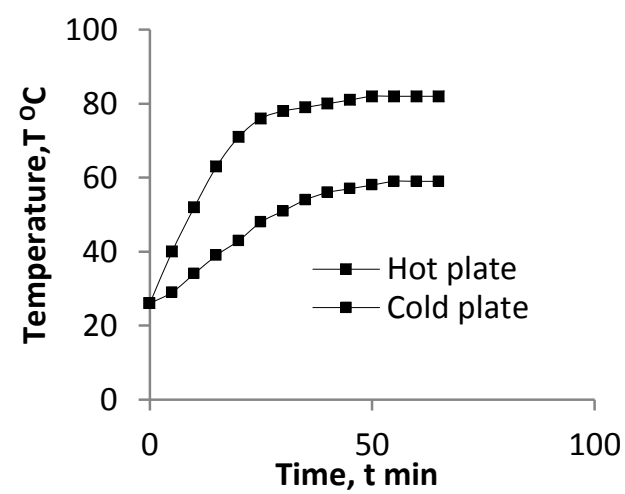

Fig.3.1: Temperature variation versus time on the upper and lower surface of a composite sample with fiber content $5-35 \mathrm{wt} \%$ and average fiber length $2 \mathrm{~mm}, 6 \mathrm{~mm}$ and $12 \mathrm{~mm}$.

As seen from the Figure, the temperature of both the surfaces of the sample gradually increases until attains a steady state value. As expected, upper surface being close to the heating source initially the temperature at this surface increases much faster than that at the lower surface. At steady state (which corresponds to $80^{\circ} \mathrm{C} T_{U s}$ and $60^{\circ} \mathrm{C} T_{L s}$ for the upper and lower surfaces of the sample), there is no accumulation of heat in any part of 
the sample, and the heat entering at the upper surface transfers through the whole thickness to the lower surface of the sample, which is in contact with lower metal disc. From this disc, the heat leaves the system through convection and radiation. For determining the heat loss from the lower surface at $60^{\circ} \mathrm{C}$ by convection and radiation, the fall of temperature of the lower disc due to heat loss by convection and radiation has been monitored in a separate experiment (Figure 3.2).

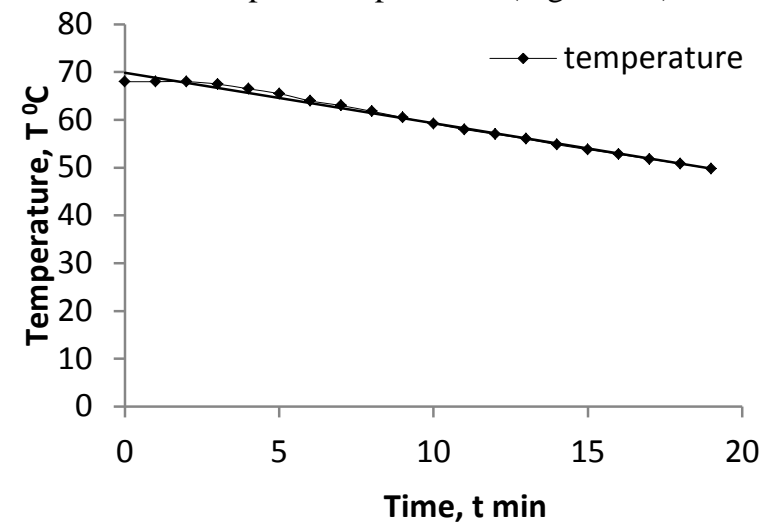

Fig.3.2. Temperature-fall pattern of the lower metal disc due to convection and radiation

The slope of the tangent drawn at $60^{\circ} \mathrm{C}$ to the curve in Figure 3.2 gives the rate of temperature fall $\left(\mathrm{dT}_{\mathrm{L}} / \mathrm{dt}\right)$ at this temperature, which multiplied ms of the disc gives, the rate of heat-loss by this disc by convection and radiation at this temperature. This value of the rate of temperature is put in the Eq. (1) to calculate the conductivity of the sample.

The thermal conductivity of the composite is presented in figure 3.3 as a function of fiber content for varying fiber length.

\subsubsection{Comparison among $2 \mathrm{~mm}, 6 \mathrm{~mm}$ and} $12 \mathrm{~mm}$ chopped jute fiber reinforced epoxy composite: As seen from the Figurw 3.3 the thermal conductivity decreases with an increase in the fiber content. Parallel to the fiber material itself, the air entrapped in the voids of the fiber contributes to the insulation of the material. The thermal conductivity of the air is $0.026[\mathrm{~W} /(\mathrm{m} . \mathrm{K})]$ at $25^{\circ} \mathrm{C}$. It has an excellent heat insulation effect. The weight percent of the air increases with increasing fiber content. Therefore, the thermal conductivity decreases with increasing fiber content [3]. The decreasing trend is the highest for the fiber length of $2 \mathrm{~mm}$. it appears that although the fibers have got random distribution, the aspect ratio (diameter/length) determines, to some extent, the mode of distribution and thus affects the thermal conductivity of the composite.

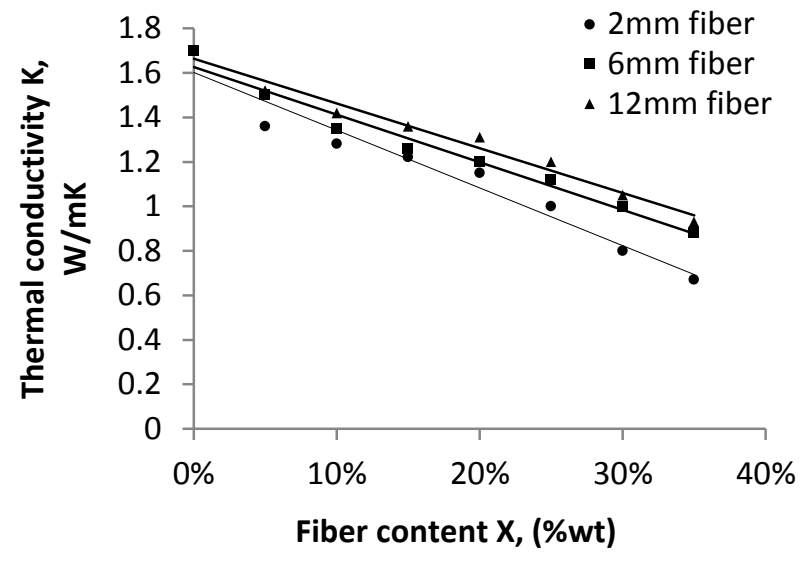

Fig. 3.3: Thermal conductivity vs. fiber content (wt $\%$ ) for $12 \mathrm{~mm}, 6 \mathrm{~mm}$ and $2 \mathrm{~mm}$ fiber length

Such trend of decrease in conductivity is reported by T.Behzad, Sain [9] for hemp fiber- reinforced acrylic polymer composite, in which the conductivity is reduced by $42.9 \%$ for the fiber content of $60 \%$ (vol). In this study, the reduction in the conductivity of the jute fiber-eopxy composite is $64.7 \%$ for fiber content of $60 \%$ (vol) (recalculated from weight $\%$ for comparison).

\subsection{Effect of fiber length and content on mechanical properties}

3.2.1. Tensile strength: The tensile strength has been plotted in Figure 3.4 as a function of fiber content for various fiber-lengths. As seen from the figure, the tensile strength decreases with the increase in the fiber content. Unlike the effect on the thermal conductivity, the fiber-length merely has any significant effect on the tensile strength observed with the range of experimentation.

Such large deterioration-rate of the strength is bit unexpected, as the epoxy resin has got good adhesion to most of the materials, but still the stress-failure could be attributed to the inadequate adhesion due to the high viscosity of the resin, which results in poor wetting of the fine fibers. With the increase in the fiber content, fiber-elements are aggregated together to prevent the access of the resin to the inter-fiber space and interrupting the continuity of the matrix structure. Some air particles might have been entrapped in the voids and adsorbed to the surface of the fiber, which also contribute to the proper contact between the resin and the fiber. Consequently, the increase in fiber content leads to the decrease in strength and the material fails mainly at the fiber-matrix interface. Beckermann et al. [18] also reported that improper adhesion hindered considerable increment of tensile strength.

Wollerdorfer and Bader [19] also concluded that the incorporation of high fiber content was limited by the incompatibility issue. 


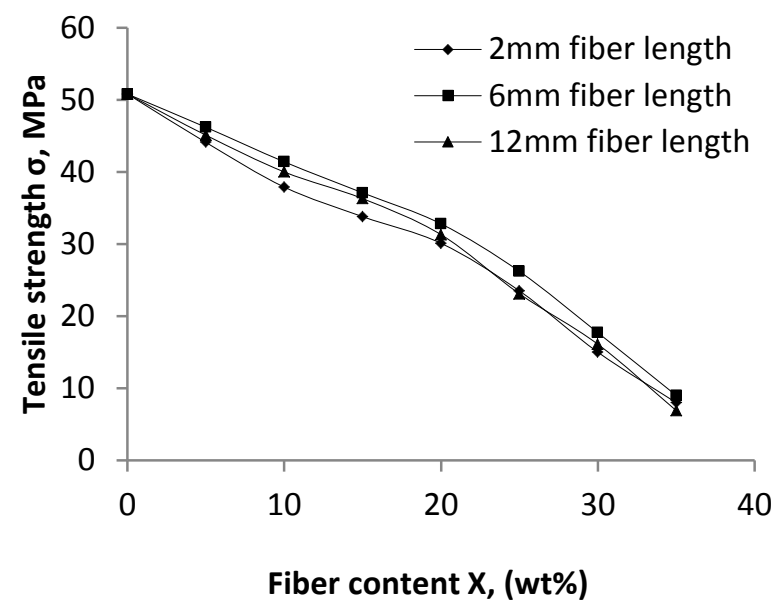

Fig. 3.4: Tensile strength of different length type fiber respect with fiber content

The bulk density of the composite decreases as the fiber content in the composition increases. To verify to what extent, the tensile strength-deterioration is compensated by the lightness of the material, the specific tensile strength, $\sigma_{s p}$ defined as the tensile strength $\sigma_{y}$ for unit specific gravity (bulk) i.e

$\sigma_{s p}=\sigma_{y} / \gamma_{b}$ is calculated and presented also in Figure 3.5 along with the bulk density.

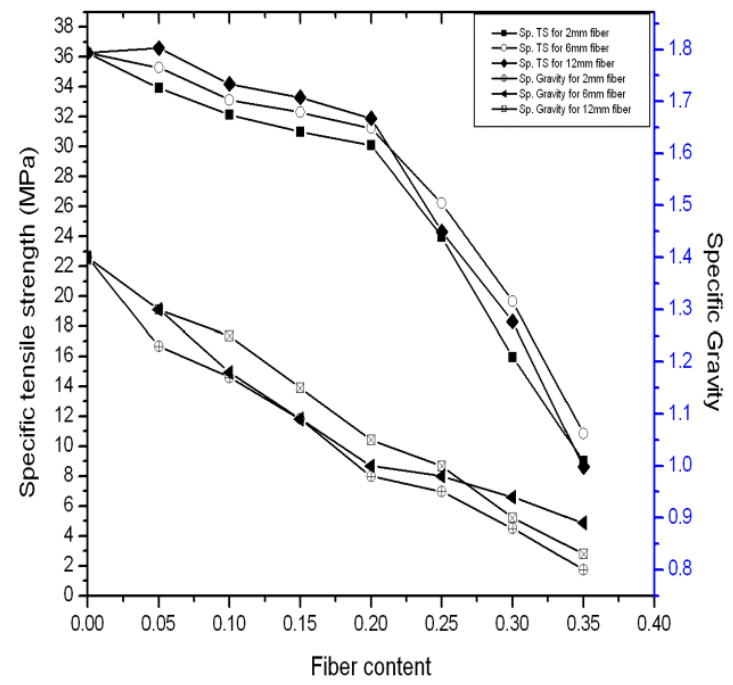

Fig. 3.5: Effect of fiber content on the bulk density and the specific tensile strength of the composite

The bulk density decreases almost linearly with the fiber content. The effect of fiber length, if exists at all, is masked within experimental errors. It is obvious that with the incorporation of the fiber in the composition, the material has become relatively lighter.

The specific tensile strength of the composite also decreases with the increase in the fiber content. Obviously the tensile strength deterioration is not compensated by the decrease in the material density.
But still the rate of strength deterioration is comparatively low up to $20 \mathrm{wt} \%$ fiber content ( $15 \%$ reduction of strength for a fiber content of $20 \mathrm{wt} \%$ ). For fiber content higher than $20 \mathrm{wt} \%$, the specific strength decreases at a much higher rate. Obviously, the non-adhesion issue is very acute and the measures should be taken to ensure good adhesion.

3.2.2. Young modulus: Young modulus is presented in Figure 3.6 as a function of fiber content. As the Figure shows, the Young modulus increases as the fiber content increases. Without further investigations, it is difficult to find explanation about why the modulus increment is the highest for the fiber length of $6 \mathrm{~mm}$ followed successively by that for the fiber length of $2 \&$ $12 \mathrm{~mm}$ violating any form of sequence of length, but the general indication is clear that the modulus increases with fiber content. At the first glance, the Young modulus data contradicts the usual perception that as the tensile strength decreases with the increase in fiber content, the Young modulus would follow similar trend. This did not happen. The reason may be that the tensile strength is measured at high stress-strain range, where the material fails at the fiber-matrix interface. On the other hand, the Young modulus is measured at low stress-strain range, where the fiber-matrix bond still withstands the applied stress and hence the modulus shows positive increment.

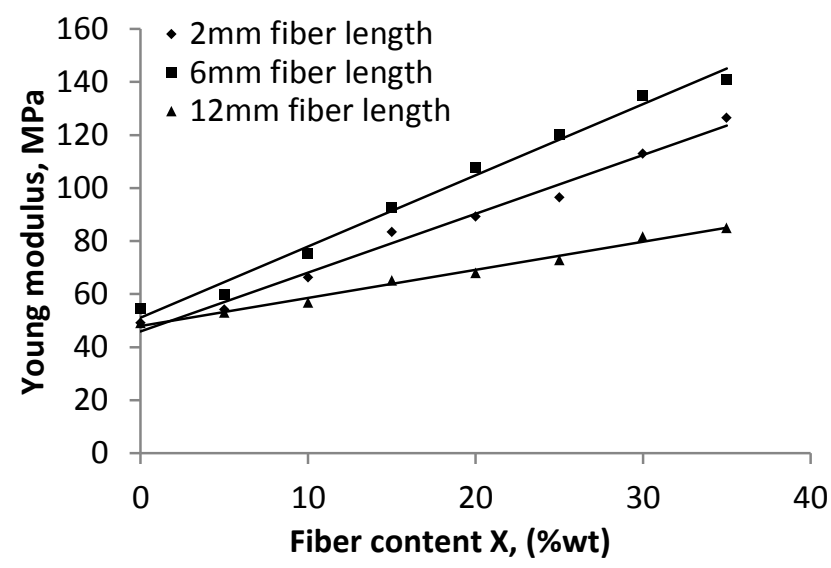

Fig. 3.6: Young modulus $E$ vs. fiber content $x$ with varying fiber length

3.2.3. Elongation at break, $\boldsymbol{\varepsilon}_{\mathbf{b}}$ : Figure 3.7 indicates that the elongation at break, $\varepsilon_{b}$ shows that for fiber content below $10 \mathrm{wt} \%$, the elongation at break does not vary much from that of pure hardened epoxy (even a slight increasing trend is observed), but beyond $10 \mathrm{wt} \%$ fiber content, the parameter decreases with the increase in fiber content. From the general knowledge in polymer science, it is expected that as the tensile strength decreases with an increase in the fiber content, the deformation property would show increasing trend. It rather is in agreement with the modulus of elasticity, which increases with the increase in the fiber content i.e the material has become brittle. Obviously, the fibermatrix adhesion is to be improved to achieve better results. 


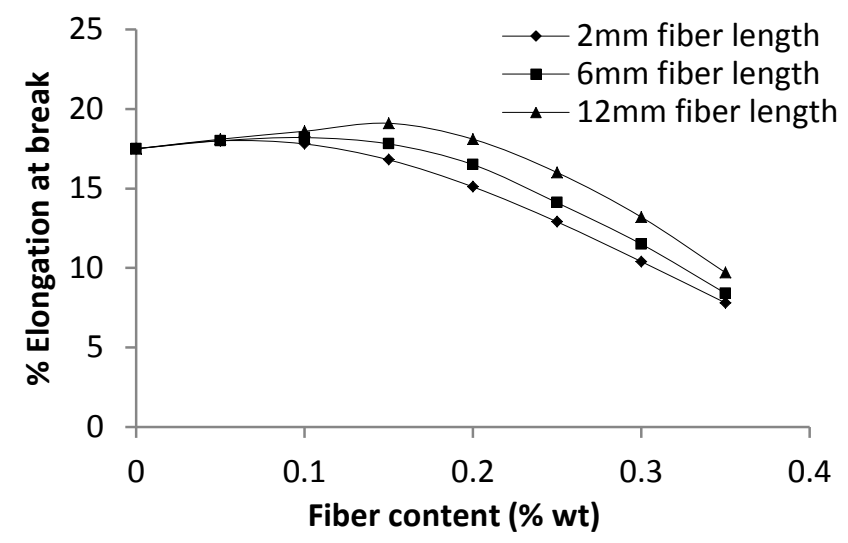

Fig. 3.7: Elongation at break, $\varepsilon_{b}$ vs. fiber content $x$ with varying fiber length

\section{CONCLUSION}

From the experimental studies, it becomes quite evident that the epoxy-jute would provide better thermal insulation that the polymer itself; but this improvement is achieved at expense of deterioration of the tensile properties of the material. More investigation is necessary to find the condition of ensuring better adhesion between the fiber and matrix in order to prevent the deterioration of the tensile properties.

From environmental viewpoint, this investigation is optimistic as it replaces non-biodegradable resin with biodegradable fiber materials. The production of resin is much more energy consuming than that of the natural fiber [21]. From socio-economical viewpoint, application of natural fiber filled polymer composite is highly inspiring as the production of resin or synthetic fiber is capital-intensive and the production of natural fiber is energy-intensive. More jobs will be created in the rural area for the cultivation and processing of natural fibers and this will contribute to the poverty alleviation program of the developing countries. Thus, more and more investigations are recommended for extending the application of natural fiber incorporated polymer composites for the thermo-, sound- and electricity insulating and vibration-dissipitating material. The TGA study is also required to ascertain the field of application of the material.

\section{REFERENCES}

[1] M. Baiardo. Z. Elisa, S. Mariastella (2004), Composite A 35 (6) 2004, pp 703-710.

[2] Li X, Tabil LG, Panigrahi S, Crerar WJ(2009), Can Biosyst Eng 2009; pp.08-148:1-10.
[3] K.John, S.Venkata Naidu, J. Rein. Plast. Comp. 26(4) (2007) 373-376.

[4] H. P. S. Abdul Khalil, S. Hanida, C. W. Kang, N.A. Nikfuaad, J. Rein. Plast. Comp. 26(2) (2007) 203-218.

[5] P.Noorunnisha Khanam, M. Mohan Reddy, K. Raghu, K. John, S. Venkata Naidu, J. Rein. Plast. Comp. 26(9) (2007) 1065-1069.

[6] S.Sreenivasulu, K. Vijay Kumar Reddy, A. Varada Rajulu, G. Ramachandra Reddy, Bull. Pure App. Sci. 25C (2) (2006) 137-142.

[7] H. Ku. and Wang, H. and Pattarachaiyakoop, N. and Trada, M. (2011 Composites Part B: Engineering, 42 (4). pp. 856-873.

[8] R. Osugi, H. Takagi, K. Liu, and Y. Gennai (2009), Proceedings of the Asian Pacific Conference for Materials and Mechanics at Yokohama, Japan, November 13-16, 2009, pp. a163_1-a163_3

[9] T .Behzad., Sain. M(2007), Published online in Wiley Inter Science.

[10] R. Mangal, N.S. Saxena, M.S. Sreekala, S. Thomas, and K. Singh, Mater. Sci. Eng., A339, 281 (2003).

[11] R.D. Sweeting and X.L. Liu, Compos. A: Appl. Sci. Manuf., 35, 933 (2004).

[12] R. Agrawal, N.S. Saxena, M.S. Sreekala, and S. Thomas, J. Polym. Sci.: Part B: Polym. Phys., 38, 916 (2000).

[13]G. Kalaprasad, P. Pradeep, G. Mathew, C. Pavithran, and S. Thomas, Compos. Sci. Technol., 60, 2967 (2000).

[14] M. Jawaid, H.P.S. Abdul Khalil, Azman Hassan, Rudi Dungani, A. Hadiyane, Composites Part B: Engineering, In Press, Corrected Proof, Available online 5 May,2012

[15] M. Jawaid, H.P.S. Abdul Khalil, A. Abu Bakar ,Materials Science and Engineering: A, Volume 527, Issues 29-30, 15 November 2010, Pages 7944-7949

[16] Jochen Gassan, Voytek S Gutowski ,Original Research Article Composites Science and Technology, Volume 60, Issue 15, November 2000, Pages 2857-2863

[17] M. Jawaid, H.P.S. Abdul Khalil, A. Abu Bakar , Original Research Article Materials Science and Engineering: A, Volume 528, Issue 15, 15 June 2011, Pages 5190-5195

[18] http://media.paisley.ac.uk/ davison/labpage/leedis $\mathrm{k} /$ leedisk.html

[19] G. W Beckermann,., Pickering, K. L. and Foreman, N. J. (2004): Materials, Proceedings of SPPM, 25-27 February 2004, Dhaka, pp. 257-265

[20] M. Wollerdorfer, and Bader, H. (1998), Industrial Crops and Products, Vol. 8,No.pp.105-1

[21] S V Joshi, Drzal L T, Mohanty A K and Arora S, Compos part A-Appl S, 35 (2004) 371 